Original Article (short paper)

\title{
Does the stretching intensity matter when targeting a range of motion gains? a randomized trial
}

\author{
Amanda de Araújo Valença ${ }^{1}$ (D), Bárbara Oliveira Soares ${ }^{1}$ (D), Bruno Remígio Cavalcante ${ }^{2}$ (D), \\ Natália Barros Beltrão ${ }^{3}$ (D), Vinicius Yan Santos Nascimento ${ }^{1,2}$ (D), \\ Ana Carolina Rodarti Pitangui ${ }^{1}$ (D), Rodrigo Cappato de Araújo ${ }^{1,2}$ (D) \\ 'Universidade de Pernambuco, Departamento de Fisioterapia, Petrolina, PE, Brasil. \\ ${ }^{2}$ Universidade de Pernambuco e Universidade Federal da Paraíba, Programa associado de pós- \\ graduação em Educação Física, Recife, PE, Brasil. \\ ${ }^{3}$ Universidade Federal Rural de Pernambuco, Departamento de Educação Física, Recife, PE, Brasil.
}

\begin{abstract}
Aims: To verify the effects of flexibility training conducted at different intensities in young adults. Methods: Twenty-one (21) young adults of both genders with no history of surgery, fracture, and/or rheumatic diseases in the lower limbs and hip, were randomly assigned to low intensity (LI) or high intensity (HI) stretching groups. Two researchers were assigned to evaluate the active knee extension range of motion (ROM) of the volunteers and two other researchers were responsible for the training program. The training consisted of a single exercise for the hamstring muscles (biceps femoris, semimembranosus, semitendinosus). Each session consisted of three repetitions of passive static stretching, maintained for the 60 s each, with 30 s interval between them, and performed three times a week for four weeks. The stretching intensity was based on the Numerical Verbal Scale, the LI group maintained the intensity between 1 and 2, while the HI group between 9 and 10. Three ROM evaluations were performed pre-intervention, after the 6th session and at the end of the 12th session. Results: No difference was observed between the groups that underwent either high- or low-intensity programs. Both groups achieved gains in flexibility after four weeks of training. Conclusions: The study demonstrated that both high- and low-intensity stretching exercises are effective for ROM and there were no differences between them. Therefore, the intensity can be defined by the preference of the therapist or patient.
\end{abstract}

Keywords: flexibility, muscle stretching exercises, youth.

\section{Introduction}

Muscle stretching exercises are part of the routines of physiotherapists and physical education professionals who aim for flexibility gains among healthy subjects or those undergoing rehabilitation ${ }^{1,2}$. Although widely practiced, there is limited evidence that supports accurate stretching prescriptions, especially regarding the intensity needed to optimize flexibility gains $s^{3,4}$.

From the studies available in the literature which tested the effect of long-period training flexibility protocols on ROM, only two analyzed the influence of different intensities established by the subjective perception of effort ${ }^{5,6}$. Both studies demonstrated that the low-intensity stretching group presented a higher range of motion (ROM) gains. However, these studies were performed with dancers, which makes it difficult to generalize the results to other populations, as individuals with high flexibility tend to present different levels of resistance and tolerance to stretching ${ }^{7}$. Muanjai et al. ${ }^{8}$ compared the effect of four weeks of stretching training at the point of dis- comfort and the point of pain. The authors did not find differences between the groups. However, as a limitation of their study the authors themselves recognized the fact that the stretches were self-administered and performed in a group session, thereby constituting aspects that may have influenced the exercise intensity control.

Thus, it is important to evaluate the intensity effect on muscle responses in other situations which are closer to the reality of clinical practice, considering subjects with limited flexibility, individualized care, and using accessible equipment to professionals. Regarding the latter aspect, most of these studies have used more robust equipment and systems for flexibility assessment, but these are often not available in clinical practice. In this context, the use of lowcost equipment with easy acquisition and handling is paramount for rehabilitation professionals. The universal goniometer is the most classically used instrument, however, the evaluator needs to have previous training in order to obtain reliable results ${ }^{9}$. On the other hand, studies have more recently highlighted the potential of using smartphone applications as an alternative tool to the goniometer 
in detriment to the low cost, availability, as well as the easy handling ${ }^{10-12}$.

Therefore, this study aimed to evaluate the effects of the passive static stretching intensity on the articular ROM of the hamstring muscles in young adults. In addition, we investigated the clinical application of two validated joint enhancement instruments, the universal goniometer, and the digital inclinometer. The study hypothesized that the stretching intensity is not a variable that has an influence on gains in flexibility.

\section{Methods}

\section{Participants}

Twenty-one (21) young adults of both genders with no history of surgery, fracture, and/or rheumatic diseases in the lower limbs and hip participated in the study. The sample calculation to determine the sample size was applied for clinical trials of parallel groups with continuous superiority outcomes ${ }^{13}$. To do so, the relative ROM gain was used as the main endpoint with values of $7.5 \%$ and $10.4 \%$ (group submitted to low intensity stretching) and 3.6\% and 6.8 (group submitted to high intensity stretching), according to values reported in Wyon et al. ${ }^{5}$ and Wyon et al. ${ }^{6}$, respectively. A minimal detectable difference of $2 \%$ for ROM was assumed ${ }^{14}$ with a two-tailed test, an alpha of 0.05 , power of 0.95 , and a loss of $20 \%$, reaching a minimum of 10 subjects per group.

The study included subjects from 18 to 22 years of age who were able to perform physical activity (identified through the Physical Activity Readiness Questionnaire PAR-Q), without training (moderate to vigorous physical activities $<150 \mathrm{~min} /$ week) for at least 30 consecutive days, presenting an active knee extension amplitude of $150^{\circ}$ or less. We excluded volunteers who became involved in some stretching or neuromuscular training program during the study protocol ${ }^{15}$. The study was approved by the Research Ethics Committee from the University under CAAE protocol (CAAE: 44949715.2.0000.5207) and all participants signed a free and informed consent form. The Clinical Trial ID for this study is RBR 46wwj7.

\section{Design and Procedures}

It is a randomized, single-blind, parallel-group randomized trial in which participants were randomly assigned to low intensity (LI) or high intensity (HI) stretching groups. Muscle stretching intensity was set as the independent variable, and knees ROM as the dependent variable.

The study was carried out at the laboratory of research of the university. The subjects' participation was voluntary, and participants were invited verbally and through social media, targeting the academic community and surrounding neighborhoods.
Group randomization occurred according to the entry order of subjects in the study through a draw of opaque envelopes which were sealed and numbered with alphanumeric code to determine the lower limb to be stretched and the stretching intensity. This code was kept confidential, and the participant was not informed to the other evaluators.

The study screening was divided into two moments and conducted by two evaluators. Anamnesis was carried out at the first moment in order to select the eligible participants through the collection of personal data, practice physical exercises and injuries, and the PAR-Q was applied. Then the anthropometric data (stature and body mass) were recorded, and the active extension angle of both knees was measured. An inclinometer - Clinometer + Bubble Level (plaincode ${ }^{\mathrm{TM}}$ ) and a universal goniometer were used for this measure.

Two researchers were assigned to evaluate ROM of the volunteers and two other researchers were responsible for the training program. The examiners were trained before the data collection began in order to guarantee the methodological rigor of the research in a pilot study. Participants were submitted to an initial evaluation (pre-intervention), an intermediate evaluation (after two weeks), and a final evaluation (four weeks).

Measurement of active knee extension ROM was initially performed with the inclinometer, positioned in the middle third of the tibia, followed by the universal goniometer measure for which the axis was positioned at the lateral condyle of the femur, the arm fixed towards the greater trochanter and the movable arm in parallel to the leg toward the lateral malleolus of the tibia. The arithmetic mean of three consecutive measurements was used as the final value. For the ROM measurements, the participants were placed on the mat in dorsal decubitus, with the contralateral lower limb held in extension and the evaluated limb at $90^{\circ}$ hip flexion maintained with the aid of an examiner.

The training consisted of a single exercise for the hamstring muscles (biceps femoris, semimembranosus, semitendinosus). Each session consisted of three repetitions of passive static stretching, maintained for $60 \mathrm{~s}$ each $^{5,6}$, with 30 s interval between them, and performed three times a week ${ }^{16}$ for four weeks. The participants' positioning was similar to that used in the evaluation but stretching proceeded passively (with feet lying on the examiner's shoulder) up to an amplitude equal to the aimed discomfort zone (intensity). The stretching intensity was based on Numerical Verbal Scale ${ }^{17}$. In this scale, the number 1 refers to a point when the subject starts to perceive pain, and 10 is the highest tolerable pain. However, we adapted the use of this scale by replacing the word "pain" with the terms tension/discomfort, as described and used by previous studies ${ }^{18,19}$. For anchoring the following instruction was given: Zero is no tension/discomfort at all 
and 10 is the worst possible tension/discomfort you could have. The LI group maintained the intensity between 1 and 2 , while the HI group between 9 and 10. We also considered that it was difficult to target in only one number, and so, we rather target a small range of numbers (1-2 and $9-10)^{19}$

The participants identified their level of discomfort every $15 \mathrm{~s}$ in order to sustain the same intensity throughout the total stretching time. Adjustments were made to the angulation when the participant reported a level of discomfort lower or higher than predicted for their group, intending to guarantee the same intensity throughout the stretching ${ }^{20}$.

\section{Statistical Analyses}

Data analysis was performed using SPSS software version 20. Mixed models were used to analyze the changes in the dependent variables in each analyzed training variation. A first-order autoregressive covariance matrix was considered. The factors of group and time were considered as fixed factors and the subjects were considered random factors. A Q-Q plot was performed for all variables to consistently and similarly confirm the normality of the residues between the observed groups. The analysis and interpretation of the within- and between-group differences were performed using mean difference values and their respective $95 \%$ confidence intervals $(95 \% \mathrm{CI})$.

\section{Results}

The recruitment of this study occurred in a single entry during the months from August to December 2018. A total of 24 people were screened for this study. Three were excluded for not meeting the eligibility criteria (Figure 1). The remaining 21 participants were evaluated at two weeks (after training) and four weeks (i.e. 10\% follow-up loss). There were only two follow-up losses, and all occurred in the group submitted to high intensity stretching.

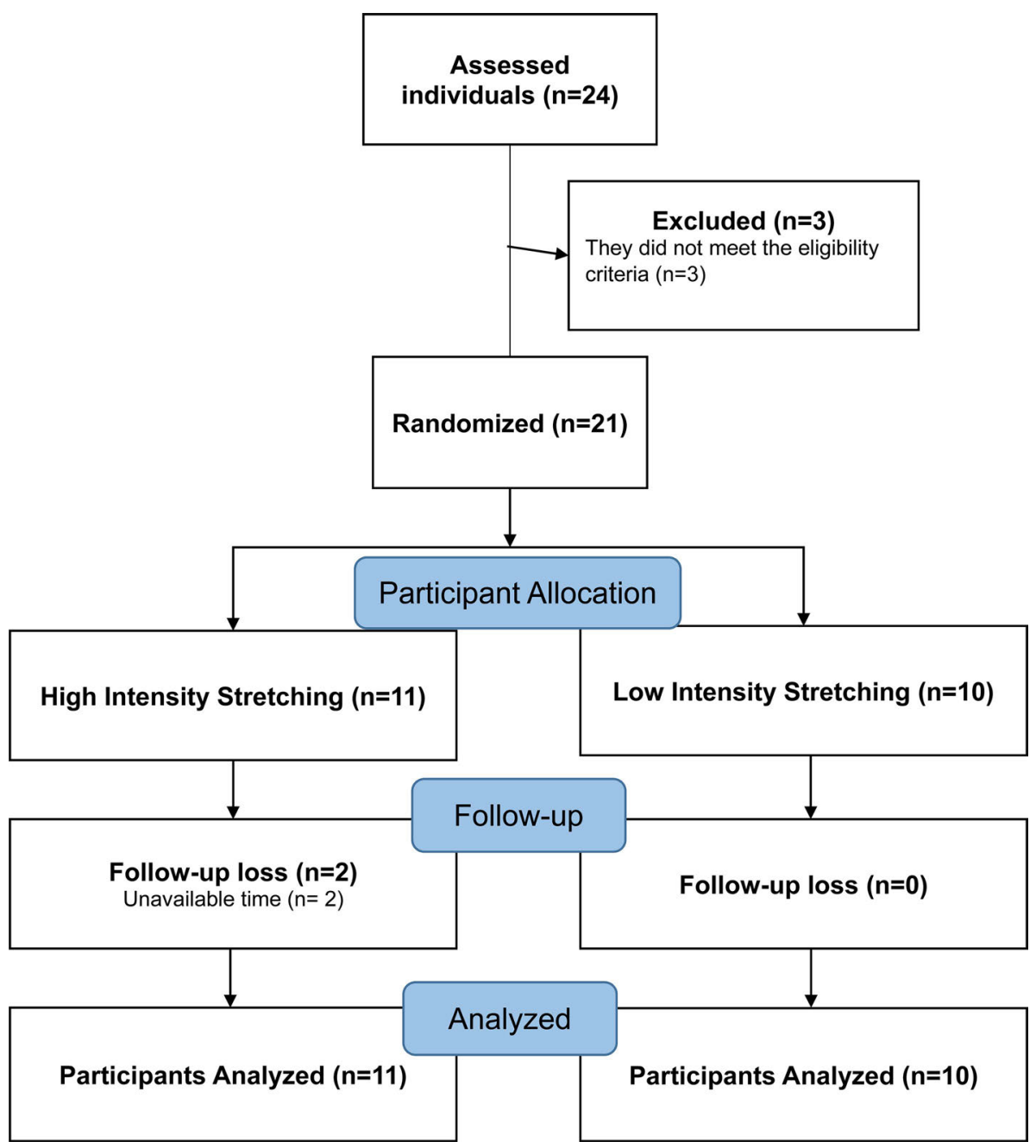

Figure 1 - Study flow diagram 
Table 1 shows the demographic characteristics of the participants in their respective groups. The groups had similar baseline characteristics.

The within-group differences showed that both groups had ROM gains in the second and fourth week of training. No significant between-group differences were observed for the ROM at four weeks. These results are presented in Table 2.

\section{Discussion}

It was possible to observe significant and similar ROM gains for the subjects who were submitted to the low and high-intensity protocols after four weeks of stretching. In addition, both goniometry and inclinometer measurements were able to record and detect similar ROM gains.

Our results confirm the findings of Muanjai et al. ${ }^{8}$, who compared the responses of subjects who trained for four weeks with stretching to the point of discomfort or the point of pain, thus being equivalent to the low and high-intensity protocol conducted herein. Both groups showed significant improvements in ROM, and there were no differences between groups. In addition to performing the ROM measurement, the passive torque responses of the groups were also evaluated, and for this measure, it was verified that the angle/torque ratio remained the same before and after the training. Based on these data, the authors discard the structural modifications and suggest that ROM improvement occurred as a result of increased tolerance by the subject to stretching.

In addition to the study by Muanjai et al. ${ }^{8}$, other evidence has suggested that the increase in muscle extensibility observed after a short-term intervention (three to eight weeks) is due to changes in the subject's sensitivity, and not to changes in muscle structure (increase in length, an increase of compliance, reduction of myotatic reflexes $)^{21,22}$. This change in sensitivity may be due to altered sensory perception or the ability of the subject to tolerate greater torque stretching ${ }^{23}$.
Some other studies with similar intervention times point to results that diverge from ours, such as the study by Wyon et al. ${ }^{5}$ and Wyon et al. ${ }^{6}$, which show higher gains for the groups subjected to more intense stretching. This divergence can be explained by the divergences in the samples between the studies. In contrast to the present study, which included young sedentary adults, the study by Wyon et al. ${ }^{5}$ and Wyon et al. ${ }^{6}$ used dancers in the sample, which tends to compromise the proximity between the studies since dancers may present different responses when the intervention is based on perceived discomfort.

Two measures were used to measure ROM in this study. The first one (the goniometer) is a low-cost instrument and has been widely used and recommended over the years. However, despite being the most frequent measure in clinical practice, reliability studies point to a great variability of intra and inter-examiner values, which vary from low to excellent ${ }^{9}$. This variation is clearly due to the procedures required for an accurate goniometry measurement, which involves handling the device, palpation of the segments and identifying anatomical points, stabilizing the segments and correct execution of the movement by the subject.

Considering goniometry limitations, the digital inclinometer was added to the study as a clinically valid and reliable alternative to assess joint ROM, being low cost and available in smartphones ${ }^{24}$. The digital inclinometer presents inter-rater reliability between moderate to very high (ICC from 0.69 to 0.98 ), and intra-rater between high to very high (ICC from 0.70 to 0.98$)^{9}$. These values reflect the simplicity of the application handling which demands little ability of the evaluator since it does not require palpation or marking anatomical points ${ }^{24,25}$.

According to the measurements gathered over the four-week stretching period, there was no difference between the ROM gains registered with the goniometer or the digital inclinometer. Thus, although absolute values were not equal (Goniometer: $\Delta \mathrm{GB}=14.2^{\circ}$ and $\Delta \mathrm{GA}=$ 16.6 $6^{\circ}$, Inclinometer: $\Delta \mathrm{GB}=12.8^{\circ}$ and $\Delta \mathrm{GA}=16.7^{\circ}$ ), both measures were sensitive to detect changes in ROM after

Table 1 - Baseline characteristics of the participants

\begin{tabular}{lccc}
\hline Characteristics & Sample & High Intensity $(\mathbf{n}=\mathbf{1 1})$ & Low Intensity $(\mathbf{n}=\mathbf{1 0})$ \\
\hline Gender & & & $5(50 \%)$ \\
$\quad$ Male n(\%) & $10(47.62 \%)$ & $5(45.5 \%)$ & $5(50 \%)$ \\
$\quad 11(52.38 \%)$ & $6(54.5 \%)$ & $19.10 \pm 1.45$ \\
$\quad$ Female n(\%) & $19.38 \pm 1.53$ & $19.63 \pm 1.63$ & $1.65 \pm 0.07$ \\
Age (years), mean (SD) & $1.65 \pm 0.79$ & $1.66 \pm 0.90$ & $63.81 \pm 14.32$ \\
Height (m), mean (SD) & $64.33 \pm 15.55$ & $64.80 \pm 17.28$ & $23.30 \pm 3.91$ \\
Weight (kg), mean (SD) & $23.24 \pm 4.20$ & $23.20 \pm 4.64$ & $142.00 \pm 11.11$ \\
BMI (kg/m $\left.{ }^{2}\right)$, mean (SD) & $141.29 \pm 13.32$ & $140.63 \pm 15.58$ & $134.00 \pm 11.10$ \\
ROM Inclinometer ( $\left.{ }^{\circ}\right)$, mean (SD) & $132.24 \pm 11.64$ & $130.63 \pm 12.42$ & \\
ROM Goniometer $\left(^{\circ}\right)$, mean (SD) & &
\end{tabular}

BMI, body mass index; ROM, range of motion. 


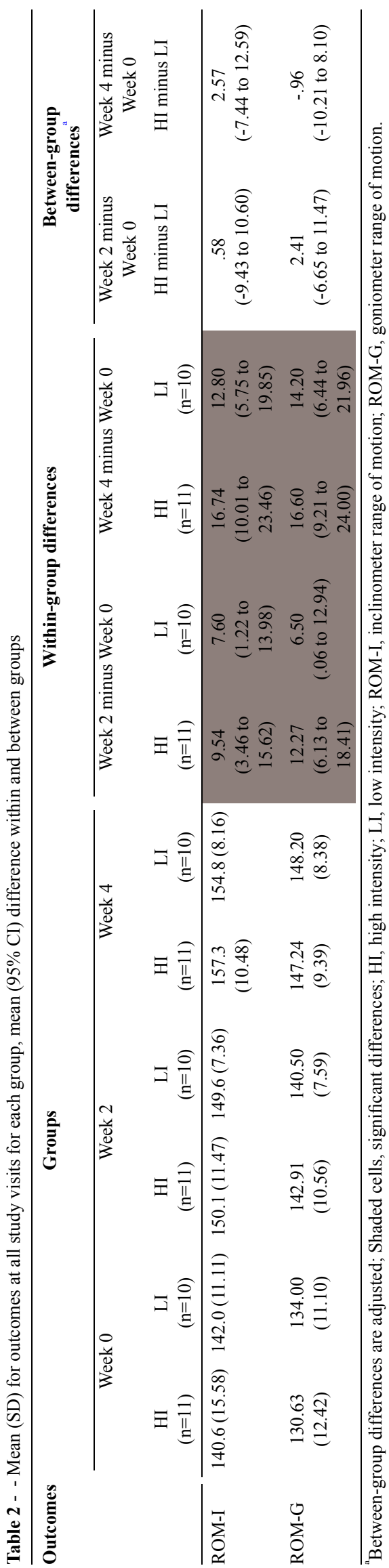

the stretching protocol. This information suggests that both devices can be used in clinical practice, provided the same instrument is used in all assessments.

This study contradicts the idea that greater gains in ROM will be achieved with intense protocols and with a high discomfort degree. The data show that a greater intensity does not imply higher gains, and that there is no need to establish the perception of pain or discomfort as a criterion to define ROM gains. As long as the stretching condition is perceived through the sensation of minimal discomfort, one can choose the amplitude of the stretching in order to make the stretching pleasant to the patient. In addition, it was found that the use of the digital inclinometer was equally efficient to measure ROM gains throughout the intervention, which encourages professionals to use a reliable, low-cost, easy access and easy manipulation resource.

The results range is limited to healthy and sedentary individuals, and therefore it cannot be extrapolated to other populations. Studies should explore the effects of different stretching intensities under other clinical conditions. In addition, it is possible that different results are observed in other time conditions and intensity parameters.

\section{Conclusion}

The results of the present study confirmed that both programs were effective in gaining flexibility, regardless of the intensity applied. Thus, the stretching intensity does not appear to be a significant variable in a four-week workout and can be defined in a shared way between therapist and patient based on individual preference.

\section{References}

1. Decoster LC, Cleland J, Altieri C, Russell P. The effects of hamstring stretching on range of motion: a systematic literature review. J Orthop Sports Phys Ther. 2005;35(6):377387.

2. Souza AC, Bentes CM, de Salles BF, Reis VM, Alves JV, Miranda H, Novaes J da S. Influence of inter-set stretching on strength, flexibility and hormonal adaptations. J Hum Kinet. 2013;36:127-135.

3. Apostolopoulos N, Metsios GS, Flouris AD, Koutedakis Y, Wyon MA. The relevance of stretch intensity and position A systematic review. Front Psychol 2015;6:1128.

4. Behm DG, Blazevich AJ, Kay AD, McHugh M. Acute effects of muscle stretching on physical performance, range of motion, and injury incidence in healthy active individuals: a systematic review. Appl Physiol Nutr Metab 2016;41(1):1-11.

5. Wyon M, Felton L, Galloway S. A comparison of 2 stretching modalities on lower-limb range of motion measurements in recreational dancers. J Strength Cond Res 2009;23(7): 2144-2148. 
6. Wyon MA, Smith A, Koutedakis Y. A comparison of strength and stretch interventions on active and passive ranges of movement in dancers: a randomized controlled trial. $\mathrm{J}$ Strength Cond Res. 2013;27(11):3053-3059.

7. Magnusson AP, Simonsen E, Aagaard P, Sørensen H, Kjaer M. A mechanism for altered flexibility in human skeletal muscle. J Physiol. 1996;497(1):291-298.

8. Muanjai P, Jones DA, Mickevicius M, Satkunskiene D, Snieckus A, Rutkauskaite R, Mickeviciene D, Kamandulis S. The effects of 4 weeks stretching training to the point of pain on flexibility and muscle tendon unit properties. Eur $\mathrm{J}$ Appl Physiol. 2017;117(8):1713-1725.

9. Santos CM dos, Ferreira G, Malacco PL, Sabino GS, Moraes GF de S, Felício DC. Intra and inter examiner reliability and measurement error of goniometer and digital inclinometer use. Rev Bras Med Esporte. 2012;18(1):38-41.

10. Jenny J-Y, Bureggah A, Diesinger Y. Measurement of the knee flexion angle with smartphone applications: Which technology is better? Knee Surg Sports Traumatol Arthrosc. 2016;24(9):2874-2877.

11. Vauclair F, Aljurayyan A, Abduljabbar FH, Barimani B, Goetti P, Houghton F, Harvey EJ, Rouleau DM. The smartphone inclinometer: a new tool to determine elbow range of motion?? Eur J Orthop Surg Traumatol. 2018;28(3):415421.

12. Youssef AR, Gumaa M. Validity and reliability of smartphone applications for clinical assessment of the neuromusculoskeletal system. Expert Rev Med Devices. 2017;14 (6):481-493.

13. Julious SA. Sample sizes for clinical trials with normal data. Statist Med. 2004;23(12):1921-1986.

14. Beltrão NB, Ritti-Dias RM, Pitangui AC, De Araújo RC. Correlation between acute and short-term changes in flexibility using two stretching techniques. Int J Sports Med. 2014;35(14):1151-1514.

15. Leite T, de Souza Teixeira A, Saavedra F, Leite RD, Rhea MR, Simao R. Influence of strength and flexibility training, combined or isolated, on strength and flexibility gains. J Strength Cond Res. 2015;29(4):1083-1088.

16. Cipriani DJ, Terry, ME, Haines MA, Tabibnia AP, Lyssanova O. Effect of stretch frequency and sex on the rate of gain and rate of loss in muscle flexibility during a hamstring-stretching program: a randomized single-blind longitudinal study. J Strength Cond Res. 2012;26(8):2119-2129.

17. Ferreira-Valente MA, Pais-Ribeiro JL, Jensen MP. Validity of four pain intensity rating scales. Pain. 2011;152 (10):2399-2404.

18. Beltrão NB, Ximenes Santos C, de Oliveira VMA, Pirauá ALT, Behm D, Pitangui ACR, de Araújo RC. Effects of a 12-week chronic stretch training program at different intensities on joint and muscle mechanical responses: a randomized clinical trial. J Sport Rehabil. 2019;24:1-9.

19. Santos CX, Beltrão NB, Pirauá ALT, Durigan JLQ, Behm $\mathrm{D}$, de Araújo RC. Static stretching intensity does not influence acute range of motion, passive torque, and muscle architecture. J Sport Rehabil. 2019;29(1):1-6.

20. Cabido CE, Bergamini JC, Andrade AG, Lima FV, Menzel HJ, Chagas MH. Acute effect of constant torque and angle stretching on range of motion, muscle passive properties, and stretch discomfort perception. J Strength Cond Res. 2014;28(4): 1050-1057.

21. Ben M, Harvey LA. Regular stretch does not increase muscle extensibility?: a randomized controlled trial. Scand J Med Sci Sports. 2010;20(1):136-144.

22. Folpp H, Deall S, Harvey LA, Gwinn T. Can apparent increases in muscle extensibility with regular stretch be explained by changes in tolerance to stretch? Aust J Physiother. 2006;52(1):45-50.

23. Weppler $\mathrm{CH}$, Magnusson SP. Increasing muscle extensibility: a matter of increasing length or modifying sensation? Physical Therapy. 2010;90(3):438-449.

24. Milanese S, Gordon S, Buettner P, Flavell C, Ruston S, Coe D, O'Sullivan W, McCormack S. Reliability and concurrent validity of knee angle measurement: smart phone app versus universal goniometer used by experienced and novice clinicians. Manual Therapy. 2014;19(6):569-574.

25. Venturini C, André A, Aguilar BP, Giacomelli B. Reliability of two evaluation methods of active range of motion in the ankle of healthy individuals. Acta Fisiatr. 2006;13(1):39-43.

\section{Corresponding author}

Vinicius Yan Santos Nascimento Department of Physical Therapy, University of Pernambuco, Petrolina, Brazil. Br 203 km 2 S/N, Cidade Universitária, 56328-903 Petrolina, PE, Brazil.

E-mail: viniciusyan@hotmail.com

Manuscript received on October 5, 2019

Manuscript accepted on March 26, 2020

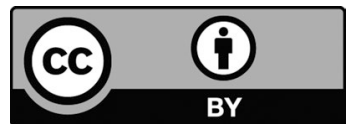

Motriz. The Journal of Physical Education. UNESP. Rio Claro, SP, Brazil - eISSN: 1980-6574 - under a license Creative Commons - Version 4.0 